\title{
Day-night cloud asymmetry prevents early oceans on Venus but not on Earth
}

Martin Turbet ${ }^{1}$, Emeline Bolmont ${ }^{1}$, Guillaume Chaverot $^{1}$, David Ehrenreich ${ }^{1}$, Jérémy Leconte ${ }^{2}$, Emmanuel Marcq $^{3}$

Earth has had oceans for nearly four billion years ${ }^{1}$ and Mars had lakes and rivers 3.5-3.8 billion years ago $^{2}$. However, it is still unknown whether water has ever condensed on the surface of Venus ${ }^{3,4}$ because the planet - now completely dry ${ }^{5}$ - has undergone global resurfacing events that obscure most of its history ${ }^{6,7}$. The conditions required for water to have initially condensed on the surface of Solar System terrestrial planets are highly uncertain, as they have so far only been studied with one-dimensional numerical climate models $^{3}$ that cannot account for the effects of atmospheric circulation and clouds, which are key climate stabilizers. Here we show using three-dimensional global climate model simulations of early Venus and Earth that water clouds - which preferentially form on the nightside, owing to the strong subsolar water vapour absorption - have a strong net warming effect that inhibits surface water condensation even at modest insolations (down to $325 \mathrm{~W} / \mathrm{m}^{2}$, that is, 0.95 times the Earth solar constant). This shows that water never condensed and that, consequently, oceans never formed on the surface of Venus. Furthermore, this shows that the formation of Earth's oceans required much lower insolation than today, which was made possible by the faint young Sun. This also implies the existence of another stability state for present-day Earth: the 'Steam Earth', with all the water from the oceans evaporated into the atmosphere.

${ }^{1}$ Observatoire astronomique de l'Université de Genève, Chemin Pegasi 51, 1290 Versoix, Switzerland. ${ }^{2}$ Laboratoire d'astrophysique de Bordeaux, Université de Bordeaux, CNRS, B18N, Allée Geoffroy Saint-Hilaire, F-33615 Pessac, France. ${ }^{3}$ LATMOS/IPSL, UVSQ, Université Paris-Saclay, Sorbonne Université, CNRS, Guyancourt, France. 
Most numerical studies that seek to identify the conditions that allow terrestrial planets to have surface liquid water (in the form of lakes, seas or oceans) assume that surface liquid water was present in the first place. Studies of Mars have focused on finding the conditions necessary to prevent complete glaciation ${ }^{8}$, in an attempt to explain widespread evidence of past intermittent hydrological activities ${ }^{2,9,10}$. Studies of Venus have focused on finding the conditions necessary to delay complete evaporation ${ }^{4,11}$. Deuterium-hydrogen $(\mathrm{D} / \mathrm{H})$ isotopic ratio measurements ${ }^{12,13}$ (about $10^{2}$ times higher than on Earth) suggest indeed that the early Venus superficial water reservoir ranged from roughly 4 to $500 \mathrm{~m}$ global equivalent layer ${ }^{4}$ (GEL; that is, the thickness of the liquid water layer if spread evenly across the surface), but could have been even higher as the $\mathrm{D} / \mathrm{H}$ fractionation factor during (hydrodynamic) escape of hydrogen can be close to unity ${ }^{14}$. The early Venus superficial water reservoir was thus much higher than today ${ }^{5}$ (about $3 \mathrm{~cm} \mathrm{GEL)} \mathrm{and} \mathrm{was} \mathrm{possibly} \mathrm{in} \mathrm{the} \mathrm{form} \mathrm{of} \mathrm{a} \mathrm{liquid} \mathrm{water}$ ocean $^{4,11,15,16}$. Studies of Earth have focused on finding the conditions necessary to prevent both complete glaciation ${ }^{17,18}$ and evaporation ${ }^{19,20}$. This is not only to match the geological record and solve the so-called faint young Sun paradox ${ }^{1,17,20,21}$, but also to predict the future of Earth $^{19,20}$. These studies have demonstrated that inherently three-dimensional (3D) atmospheric processes are key stabilizers for maintaining a surface liquid water ocean. Of particular interest for this study, the slow rotation of Venus could lead to the formation of subsolar clouds, which would effectively reflect the incident solar flux and thus stabilize oceans ${ }^{4}$ (Fig. 1a). On fastrotating planets like Earth, atmospheric dynamics produce dry regions (in the descending branches of atmospheric cells, for example, Hadley cells) that would increase thermal emission to space, cooling and thus stabilizing oceans ${ }^{19,22}$.

However, even before the question of the conditions for maintaining a surface liquid water ocean arises, water initially present in the young and warm planetary atmosphere must be able to condense on the surface. Planets are indeed expected to form hot due to their initial

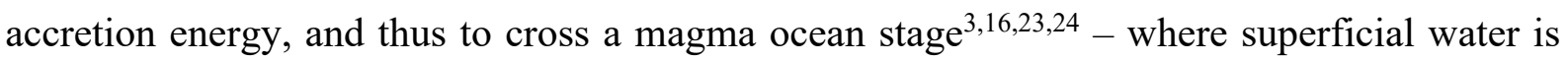
present only in the form of vapour - before evolving towards their final state. The conditions leading to the condensation of a water ocean after the magma ocean phase have so far been studied with only one-dimensional (1D) numerical climate models ${ }^{3,24}$, which neglect the effects of atmospheric dynamics and clouds. Hot, water-vapour dominated atmospheres are indeed 
notoriously difficult ${ }^{19}$ to simulate in three dimensions because they require an adaptation of condensation, convection, radiation and convergence schemes in a regime where water vapour can condense and yet be the dominant gas.

Here we perform 3D global climate model (GCM) simulations designed to simulate the set of conditions required for water condensation and, consequently, ocean formation to take place on terrestrial planets, and in particular on early Venus and Earth. For this, we adapted the GCM to cope with the extreme environments of hot, water-dominated primitive atmospheres, as well as their extremely long convergence time (see Methods). Simulations of initially hot (initial temperature around $\left.10^{3} \mathrm{~K}\right)$ and water vapour-dominated $\left(\mathrm{H}_{2} \mathrm{O}\right.$ partial pressure $10 \mathrm{bar}$ ) atmospheres of early Venus and Earth (forced to insolations between 300 and $675 \mathrm{~W} / \mathrm{m}^{2}$, defined here as average top-of-the-atmosphere fluxes) reveal that clouds are essentially concentrated on the nightside (Fig. 2a, b, e, f), independently of the planetary rotation speed and day duration. The absence of clouds and strong solar absorption by water vapour on the dayside reduce the planetary albedo. The presence of clouds on the nightside produces a strong greenhouse warming, which effectively reduces the nightside thermal cooling to space (Fig. 2c, d, g, h). As a result, clouds have a strong net warming effect (Extended Data Fig. 2).

Preferential nightside water cloud formation is mainly driven by the direct visible and near-infrared absorption by water vapour - here the dominant gas - of the incoming solar radiation. First, the absorption by water vapour produces a strong atmospheric heating in the subsolar region - which has already been qualitatively observed ${ }^{25,26}$ - that efficiently warms the atmosphere down to about 1 bar (Fig. 3b), which breaks moist convection, and therefore strongly inhibits dayside cloud formation. The dayside cloud cover in fact decreases with increasing incident flux, as illustrated qualitatively in Fig. 2a, b, e, f and quantitatively in Extended Data Fig. 3b. For high insolation (that is, about $650 \mathrm{~W} / \mathrm{m}^{2}$, which is equivalent to the solar flux on present-day Venus), the dayside is almost entirely depleted in clouds, and the bond albedo reaches closely that of previous state-of-the-art 1D numerical climate model cloud-free calculations $^{27-29}$ (Methods). Figure 3d in fact shows that this direct subsolar absorption is up to about $10^{4}$ stronger than in standard 3D GCM simulations where all water is initially assumed to be condensed on the surface (labelled 'cold start' in Fig. 3). This is due to the fact that the 
water vapour mixing ratio is up to about $10^{7}$ larger, at least in the upper part of the atmosphere (Fig. 3c).

In our baseline simulations of hot and steamy Earth and Venus, the atmospheric circulation is strongest in the upper atmosphere (above about 0.1 bar). It is dominated by a strong eastward jet that is even super-rotating in the early Venus simulations, owing to the slower planet rotation. It is also characterized by a Brewer-Dobson-like circulation (Fig. 3a) which transports warm stratospheric air parcels from the equator to the poles. As the warm and dry stratospheric equatorial air parcels get transported eastward and poleward by the winds, they cool by thermal infrared emission, which eventually leads to preferential cloud formation on the nightside, specifically at two gyres, and at the poles (Fig. 2a, b, e, f), where the balance between thermal infrared cooling and dynamical heat redistribution heating (by the winds) is favourable. The mechanism leading to the preferential formation of clouds in the nightside is depicted in Fig. 1b. The accumulation of clouds on the nightside reduces the thermal cooling to space. Figure $2 \mathrm{c}, \mathrm{d}, \mathrm{g}$, h shows that the thermal emission to space is actually anticorrelated with the position of water clouds, which produce a strong greenhouse effect. This peculiar distribution of clouds produces a strong net warming effect, between 50 and $120 \mathrm{~W} / \mathrm{m}^{2}$ in the range of insolation of around 340-675 W/m², that is, that of Earth and Venus (Extended Data Fig. 2). At high insolations, nightside temperatures increase with insolation, resulting in a reduction in cloud cover and thus in greenhouse warming of clouds.

The nightside cloud pattern and its net warming effect are robust to a wide range of insolations (Fig. 2). They are also robust to a wide range of cloud properties, total amount of water vapour and addition of carbon dioxide $\left(\mathrm{CO}_{2}\right)$ (Methods). The preferential formation of stratospheric nightside water clouds in both the Earth (sidereal rotation period $P_{\text {rot }} \sim 24 \mathrm{~h}$ ) and early Venus simulations ( $P_{r o t} \sim 5,833 \mathrm{~h}$, that is, about 244 times that of Earth) suggests that the mechanism is also robust to a wide range of rotation periods. A comprehensive sensitivity study is needed to quantitatively confirm this result, as it has been shown that cloud and atmospheric circulation feedbacks can vary nonlinearly and non-monotonically with rotation period ${ }^{30}$. The mechanism differs from the stabilizing tropospheric subsolar cloud feedback ${ }^{4,31}$ (Fig. 1a), which works only for slowly-rotating planets ${ }^{31-33}$. This is because the stratosphere adjusts here quickly to the solar heating perturbation (constantly destroying clouds on the dayside and reforming 
them on the nightside) compared with the troposphere, which requires a much longer daylight period to establish a region of stable and intense moist convection, capable of producing thick, reflective clouds ${ }^{32}$.

Cloud formation in the GCM is largely dominated by large-scale condensation (that is, water vapour condensation driven by large-scale air movements, as illustrated in Fig. 3a) and not by subgrid-scale condensation (driven by small convective cells, as in effs $^{4,31}$ ). This indicates that our results should be weakly sensitive to the choice of the subgrid moist convection parameterization, which is a strong indication ${ }^{34}$ that preferential nightside cloud formation is likely to be a robust mechanism across GCMs.

As a direct consequence, the net warming effect of clouds markedly reduces the insolation required to condense water on the surface, compared with previous 1D cloud-free calculations ${ }^{3,16,24}$. The simulations we have carried out at multiple insolations reveal that (1) the insolation required to condense water on early Venus is about $325 \mathrm{~W} / \mathrm{m}^{2}$ (or about 0.95 times the Earth solar constant) and (2) that on Earth is about $312.5 \mathrm{~W} / \mathrm{m}^{2}$ (or about 0.92 times the Earth solar constant). When the initially hot and steamy planetary atmospheres reach these insolation thresholds, clouds start to form on the dayside, which makes the bond albedo suddenly jump, and produce a net cooling of the atmosphere until water vapour starts to condense on the surface, forming oceans (see an example for early Venus in Extended Data Fig. 1, $325 \mathrm{~W} / \mathrm{m}^{2}$ ). This behaviour is visible through evaluations of the bond albedo (red and blue curves in Extended Data Fig. 3b) as well as the amount of reflected incoming solar radiation (red curves in Extended Data Fig. 2) which both substantially increase as the insolation approaches the water condensation insolation thresholds. This implies that a young and therefore hot terrestrial planet can form oceans on its surface only if the insolation that it receives is lower than the aforementioned insolation thresholds, as illustrated in Fig. 4 (red branches). States of stability where surface oceans are present (Fig. 4, blue branches) are possible only if (1) most water is initially condensed on the surface and (2) the insolation is below that of the runaway greenhouse $e^{4,19}$. The fact that the water condensation insolation threshold is low (owing to the nightside cloud feedback presented in this study) compared with the runaway greenhouse insolation threshold (which is higher, due to the dayside cloud feedback on Venus ${ }^{4}$ and the dry descending branches of the Hadley cell on Earth ${ }^{19}$ ) produces a 
hysteresis loop with two possible stability states (Fig. 4), with water either fully condensed on the surface (blue branches) or fully evaporated (red branches) in the atmosphere.

These results have important consequences for our understanding of the comparative evolution of the atmosphere of Earth and Venus, as well as of rocky exoplanets. The minimal insolation received by Venus in the course of its evolution is about $500 \mathrm{~W} / \mathrm{m}^{2}$ (or about 1.47 times the Earth solar constant and about 0.75 times the Venus solar constant, around 4 billion years ago). As this minimal insolation is much higher than the water condensation insolation threshold (about $325 \mathrm{~W} / \mathrm{m}^{2}$ ), oceans should never have formed on the surface of Venus, which confirms and extends previous 1D cloud-free climate predictions ${ }^{3}$. Sensitivity studies show that this result is robust to a wide range of water and $\mathrm{CO}_{2}$ contents (Methods). The water above the surface of Venus - always in the form of vapour - most probably gradually escaped from the atmosphere. It is not possible to tell from $\mathrm{D} / \mathrm{H}$ measurements alone ${ }^{35-37}$ whether this scenario is more likely than the one in which a late ocean was present ${ }^{11}$. However, recent estimates of water delivery ${ }^{38}$ and atmospheric escape calculations ${ }^{38,39}$ together suggest that water escaped very early from the atmosphere of Venus when the Sun's extreme ultraviolet radiation was stronger ${ }^{40,41}$, in better agreement with our proposed scenario.

The insolation received on Earth today (about $340.5 \mathrm{~W} / \mathrm{m}^{2}$ ) is higher than the water condensation insolation threshold (about $312.5 \mathrm{~W} / \mathrm{m}^{2}$ ). It means first - as initially suggested with simplified 1D cloud-free climate models ${ }^{28}$ - that the present-day Earth possesses three main stability states: (1) the present-day Earth with surface liquid oceans; (2) the snowball Earth $^{42}$ with oceans (quasi-) completely frozen; and (3) the steam Earth (this study), with water mostly in the form of steam. It also means that the reduced past solar luminosity (about $75 \%$ fainter 4 billion years ago) had a key role in allowing water to condense on Earth in the first place (by lowering the solar flux received on Earth below the water condensation insolation threshold) and form oceans, and thus for the emergence and development of life on Earth. This suggests that the faint young Sun, which has long been problematic in explaining the longevity of the Earth's liquid water oceans ${ }^{21}$, may in fact have been a necessary opportunity for life to appear on Earth. 


\section{REFERENCES}

1. Catling, D. C. \& Zahnle, K. J. The Archean atmosphere. Science Advances 6, eaax 1420 (2020).

2. Haberle, R. M., Catling, D. C., Carr, M. H. \& Zahnle, K. J. The Early Mars Climate System, 526-568. Cambridge Planetary Science (Cambridge University Press, 2017).

3. Hamano, K., Abe, Y. \& Genda, H. Emergence of two types of terrestrial planet on solidification of magma ocean. Nature 497, 607-610 (2013).

4. Way, M. J. et al. Was Venus the first habitable world of our solar system? Geophysical Research Letters 43, 8376-8383 (2016).

5. Bézard, B. \& de Bergh, C. Composition of the atmosphere of Venus below the clouds. Journal of Geophysical Research (Planets) 112, E04S07 (2007).

6. Phillips, R. J. et al. Impact Craters and Venus Resurfacing History. Journal of Geophysical Research 97, 15923-15948 (1992).

7. Kreslavsky, M. A., Ivanov, M. A. \& Head, J. W. The resurfacing history of Venus: Constraints from buffered crater densities. Icarus 250, 438-450 (2015).

8. Wordsworth, R. D., Kerber, L., Pierrehumbert, R. T., Forget, F. \& Head, J. W. Comparison of "warm and wet" and "cold and icy" scenarios for early Mars in a 3-D climate model. Journal of Geophysical Research (Planets) 120, 1201-1219 (2015).

9. Wordsworth, R. D. The Climate of Early Mars. Annual Review of Earth and Planetary Sciences 44, 381-408 (2016).

10. Kite, E. S. Geologic Constraints on Early Mars Climate. Space Science Reviews 215, 10 (2019).

11. Way, M. J. \& Del Genio, A. D. Venusian Habitable Climate Scenarios: Modeling Venus Through Time and Applications to Slowly Rotating Venus-Like Exoplanets. Journal of Geophysical Research (Planets) 125, e06276 (2020).

12. de Bergh, C. et al. Deuterium on Venus: Observations from Earth. Science 251, 547-549 (1991).

13. Marcq, E., Mills, F. P., Parkinson, C. D. \& Vandaele, A. C. Composition and Chemistry of the Neutral Atmosphere of Venus. Space Science Reviews 214, 10 (2018).

14. Kasting, J. F. \& Pollack, J. B. Loss of water from Venus. I. Hydrodynamic escape of hydrogen. Icarus 53, 479-508 (1983).

15. Kasting, J. F. Runaway and moist greenhouse atmospheres and the evolution of Earth and Venus. Icarus 74, 472-494 (1988).

16. Salvador, A. et al. The relative influence of $\mathrm{H}_{2} \mathrm{O}$ and $\mathrm{CO}_{2}$ on the primitive surface conditions and evolution of rocky planets. Journal of Geophysical Research (Planets) 122, 1458-1486 (2017).

17. Charnay, B. et al. Exploring the faint young Sun problem and the possible climates of the Archean Earth with a 3-D GCM. Journal of Geophysical Research (Atmospheres) 118, 10,414-10,431 (2013).

18. Wolf, E.T. \& Toon, E.B. Hospitable Archean Climates Simulated by a General Circulation Model. Astrobiology 13, 656-673 (2013).

19. Leconte, J., Forget, F., Charnay, B., Wordsworth, R. \& Pottier, A. Increased insolation threshold for runaway greenhouse processes on Earth-like planets. Nature 504, 268-280 (2013). 
20. Wolf, E. T. \& Toon, O. B. Delayed onset of runaway and moist greenhouse climates for Earth. Geophysical Research Letters 41, 167-172 (2014).

21. Charnay, B., Wolf, E. T., Marty, B. \& Forget, F. Is the Faint Young Sun Problem for Earth Solved? Space Science Reviews 216, 90 (2020).

22. Pierrehumbert, R. T. Thermostats, Radiator Fins, and the Local Runaway Greenhouse. Journal of Atmospheric Sciences 52, 1784-1806 (1995).

23. Elkins-Tanton, L. T. Linked magma ocean solidification and atmospheric growth for Earth and Mars. Earth and Planetary Science Letters 271, 181-191 (2008).

24. Lebrun, T. et al. Thermal evolution of an early magma ocean in interaction with the atmosphere. Journal of Geophysical Research (Planets) 118, 1155-1176 (2013).

25. Kopparapu, R. K. et al. Habitable Moist Atmospheres on Terrestrial Planets near the Inner Edge of the Habitable Zone around M Dwarfs. The Astrophysical Journal 845, 5 (2017).

26. Fujii, Y., Del Genio, A. D. \& Amundsen, D. S. NIR-driven Moist Upper Atmospheres of Synchronously Rotating Temperate Terrestrial Exoplanets. The Astrophysical Journal 848, 100 (2017).

27. Kopparapu, R. K. et al. Habitable Zones around Main-sequence Stars: New Estimates. The Astrophysical Journal 765, 131 (2013).

28. Goldblatt, C., Robinson, T. D., Zahnle, K. J. \& Crisp, D. Low simulated radiation limit for runaway greenhouse climates. Nature Geoscience 6, 661-667 (2013).

29. Turbet, M., Ehrenreich, D., Lovis, C., Bolmont, E. \& Fauchez, T. The runaway greenhouse radius inflation effect - an observational diagnostic to probe water on earthsized planets and test the habitable zone concept. Astronomy \& Astrophysics 628, A12 (2019).

30. Jansen, T., Scharf, C., Way, M. \& Del Genio, A. Climates of Warm Earth-like Planets. II. Rotational "Goldilocks" Zones for Fractional Habitability and Silicate Weathering. The Astrophysical Journal 875, 79 (2019).

31. Yang, J., Cowan, N. B. \& Abbot, D. S. Stabilizing Cloud Feedback Dramatically Expands the Habitable Zone of Tidally Locked Planets. The Astrophysical Journal Letters 771, L45 (2013).

32. Yang, J., Boué, G., Fabrycky, D. C. \& Abbot, D. S. Strong Dependence of the Inner Edge of the Habitable Zone on Planetary Rotation Rate. The Astrophysical Journal Letters 787, L2 (2014).

33. Kopparapu, R. K. et al. The Inner Edge of the Habitable Zone for Synchronously Rotating Planets around Low-mass Stars Using General Circulation Models. The Astrophysical Journal 819, 84 (2016).

34. Fauchez, T. J. et al. TRAPPIST Habitable Atmosphere Intercomparison (THAI) workshop report. arXiv e-prints arXiv:2104.01091. The Planetary Science Journal, in press (2021).

35. Grinspoon, D. H. Was Venus Wet? Deuterium Reconsidered. Science 238, 1702-1704 (1987).

36. Grinspoon, D. H. Implications of the high $\mathrm{D} / \mathrm{H}$ ratio for the sources of water in Venus' atmosphere. Nature 363, 428-431 (1993).

37. Gurwell, M. A. Evolution of deuterium on Venus. Nature 378, 22-23 (1995).

38. Gillmann, C. et al. Dry late accretion inferred from Venus's coupled atmosphere and internal evolution. Nature Geoscience 13, 265-269 (2020).

39. Persson, M. et al. The Venusian Atmospheric Oxygen Ion Escape: Extrapolation to the Early Solar System. Journal of Geophysical Research (Planets) 125, e06336 (2020). 
40. Lichtenegger, H. I. M et al. Solar XUV and ENA-driven water loss from early Venus' steam atmosphere. Journal of Geophysical Research (Space Physics) 121, 4718-4732 (2016).

41. Lammer, H. et al. Origin and evolution of the atmospheres of early Venus, Earth and Mars. The Astronomy and Astrophysics Review 26, 2 (2018).

42. Hoffman, P. F., Kaufman, A. J., Halverson, G. P. \& Schrag, D. P. A Neoproterozoic snowball Earth. Science 281, 1342-1346 (1998).

43. Shields, A. L., Bitz, C. M., Meadows, V. S., Joshi, M. M. \& Robinson, T. D. Spectrumdriven Planetary Deglaciation due to Increases in Stellar Luminosity. The Astrophysical Journal Letters 785, L9 (2014). 


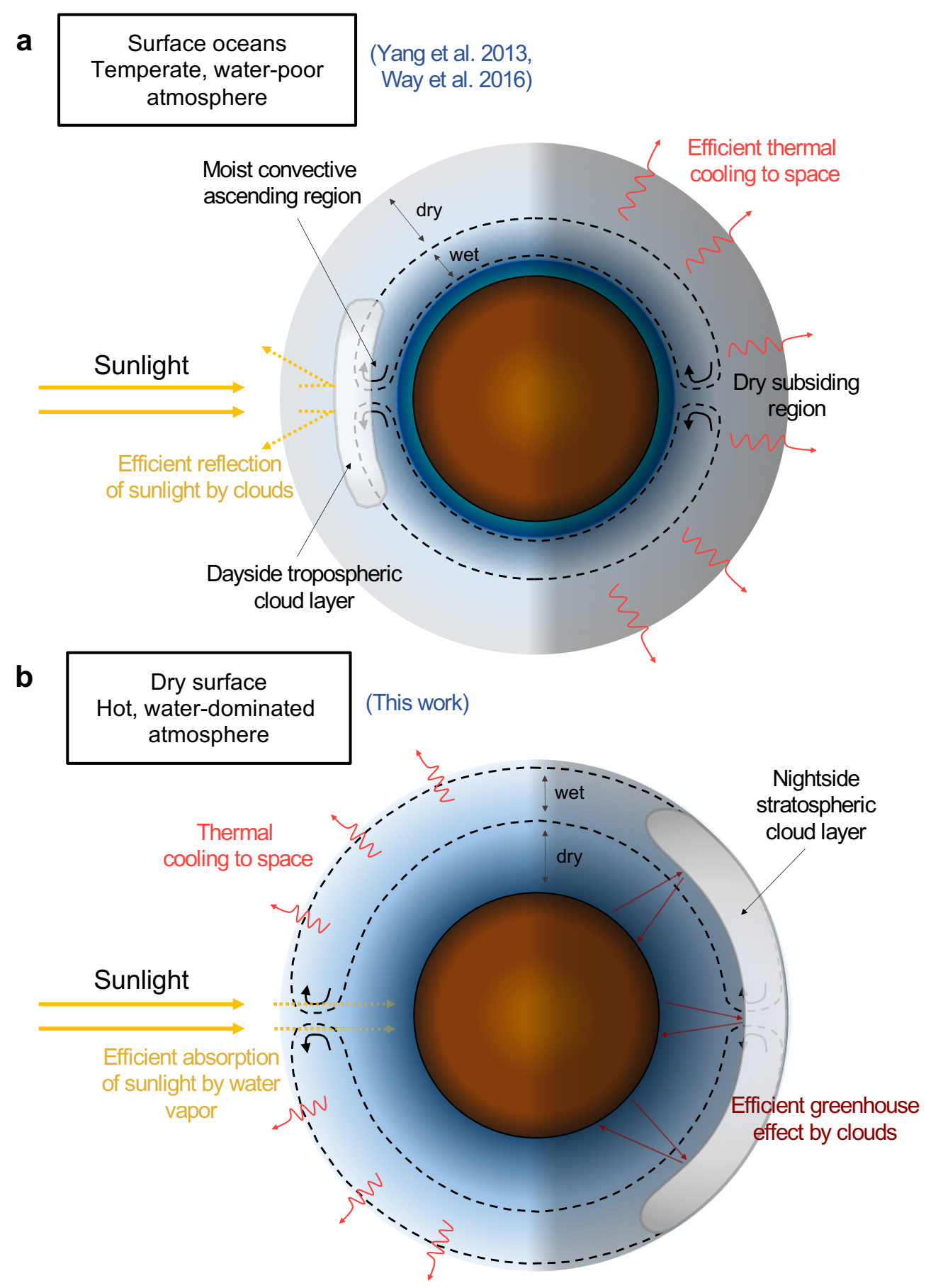

Figure 1 | Day and night water cloud feedbacks. a,b, Sketches of the cloud feedback mechanisms on slowly rotating planets initially covered with a liquid water $\operatorname{ocean}^{4,31}$ (a) and fast and slowly rotating planets with oceans initially completely evaporated into the atmosphere (this study) (b). Depending on their position (dayside or nightside), clouds have a very strong net cooling (a) or warming (b) effect. 

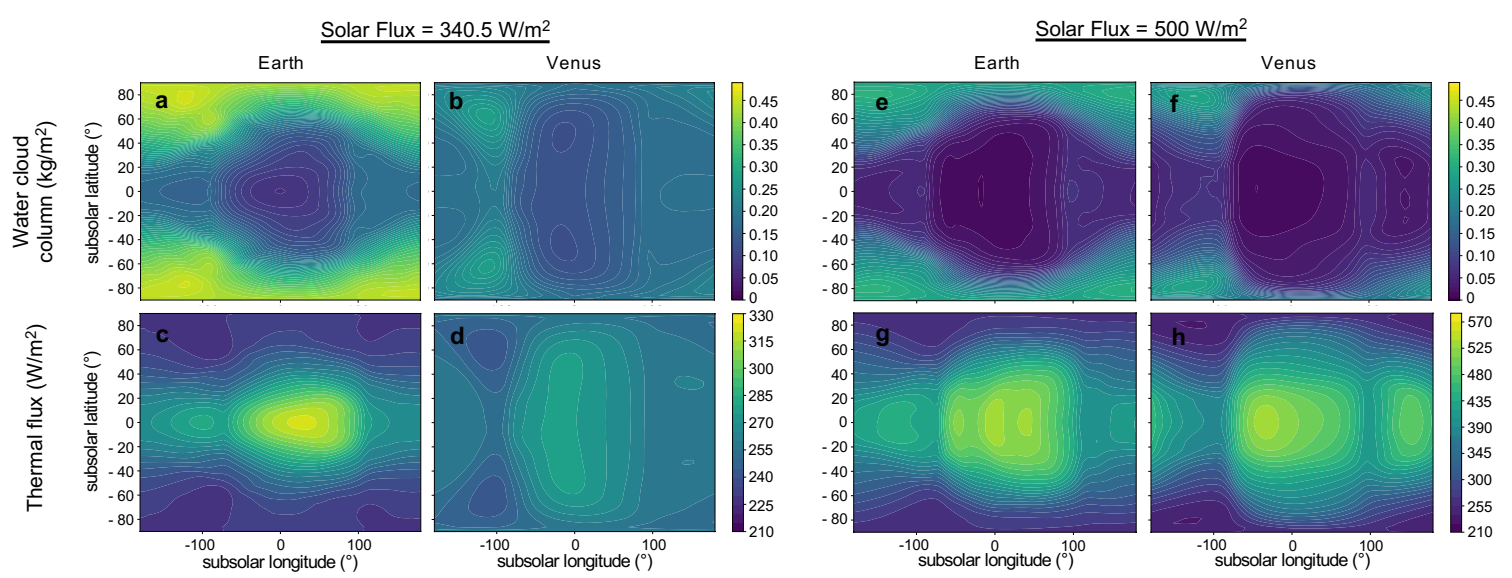

Figure 2 | Water clouds and thermal emission horizontal maps. a-h Maps of water cloud column $(\mathbf{a}, \mathbf{b}, \mathbf{e}, \mathbf{f})$ and thermal emission to space $(\mathbf{c}, \mathbf{d}, \mathbf{g}, \mathbf{h})$ for initially hot and steamy Earth $\left(P_{r o t} \sim 24 \mathrm{~h} ; \mathbf{a}, \mathbf{c}, \mathbf{e}, \mathbf{g}\right)$ and Venus $\left(P_{r o t} \sim 5,833 \mathrm{~h}\right.$, that is, 244 times that of Earth; b, d, f, h), forced to insolations of $340.5 \mathrm{~W} / \mathrm{m}^{2}$ (that is, the present-day Earth insolation; a-d) and 500 $\mathrm{W} / \mathrm{m}^{2}$ (that is, the minimal insolation received on Venus, about 4 billion years ago when the Sun was $25 \%$ fainter than today; e-h). The maps were calculated in the heliocentric frame (that is, keeping the subsolar point at $0^{\circ}$ longitude and $0^{\circ}$ latitude), and using an average of five Earth years (for Earth simulations) and five Venusian days (for Venus simulations), respectively. In all simulations, clouds are preferentially located on the nightside, which markedly reduces the thermal emission to space. 

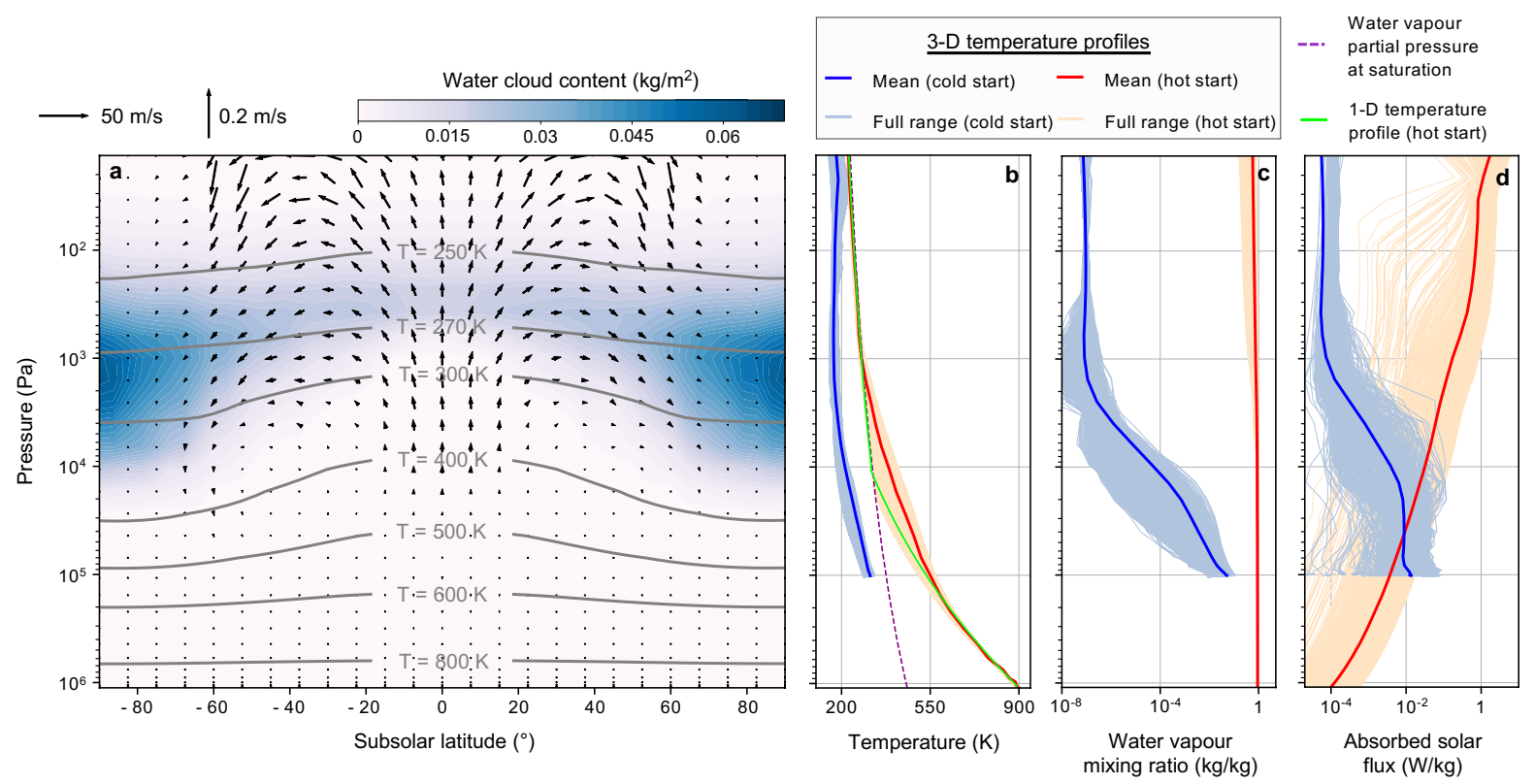

Figure 3 | Mechanisms of nocturnal cloud formation. (a) Annual-mean average zonal water cloud distribution (in the heliocentric frame) for initially hot and steamy Earth, forced at an insolation of $340.5 \mathrm{~W} / \mathrm{m}^{2}$, on which atmospheric temperature contours and local wind field have been superimposed. (b-d) Vertical profiles of the annual-mean globally averaged atmospheric temperatures (b), water vapour mixing ratios (c) and absorbed solar flux (d). The red vertical profiles were computed using the baseline simulation of hot and steamy Earth $\left(340.5 \mathrm{~W} / \mathrm{m}^{2}\right.$, $\mathrm{P}_{\mathrm{H} 2 \mathrm{O}}=10$ bar: 'hot start'). The blue vertical profiles were computed for present-day Earth (aquaplanet) conditions, with all water initially condensed on the surface ('cold start'). The green temperature profile was computed using a $1 \mathrm{D}$ reverse radiative-convective mode ${ }^{29}$ of hot and steamy Earth as in refs ${ }^{27,28}$. The water vapour condensation curve (dotted purple) was calculated for the water vapour mixing ratio $(0.91)$ of the warm and steamy simulations. Solar heating on the dayside pushes the tropopause downward so that clouds are formed by large scale radiative cooling of high-altitude air masses moving towards the nightside. 


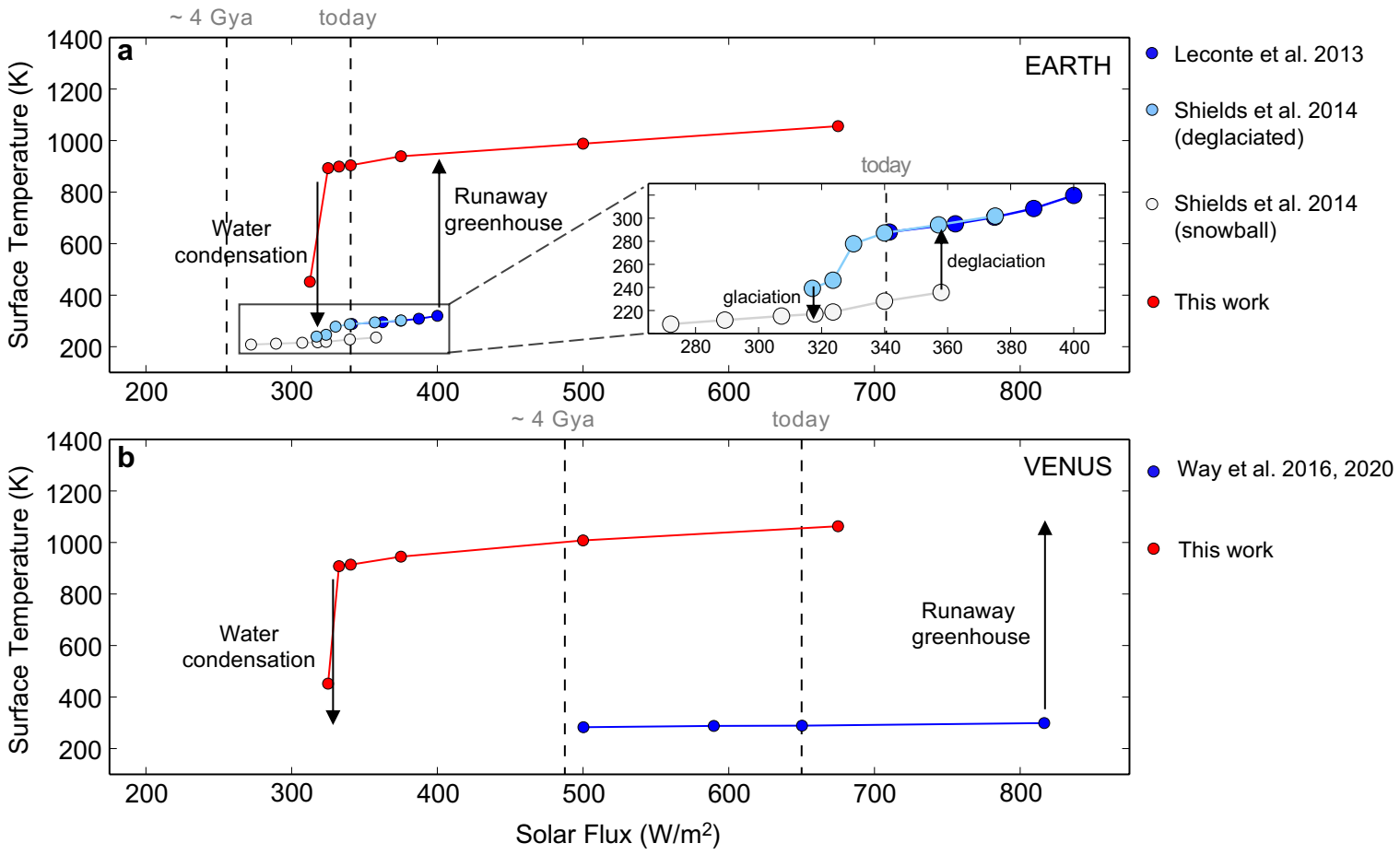

Figure 4 | Hysteresis loops and conditions of ocean formation on Earth and Venus. a,b, Surface temperature of Earth (a) and Venus (b) as a function of incoming solar flux. The blue branch corresponds to simulation results ${ }^{4,11,19,43}$ that assume water is initially condensed on the surface. The red branch (this study) corresponds to initially hot and steamy simulation results, that assume water is initially in vapour form in the atmosphere. For reference, insolation is $340.5 \mathrm{~W} / \mathrm{m}^{2}$ on present-day Earth and is $650 \mathrm{~W} / \mathrm{m}^{2}$ on present-day Venus. Insolations were $25 \%$ lower about 4 billion years ago (Ga). Although the insolation on Earth 4 billion years ago was low enough for it to reach the blue branch and thus form surface oceans, the insolation on initially hot and steamy Venus forced it directly into the red branch, preventing surface water condensation. 


\section{METHODS}

\section{Numerical climate model and simulations setup}

The simulations were performed with the LMD Generic Global Climate Model (GCM). The model, which was historically developed to study the climate of the Earth ${ }^{44}$ and then Mars ${ }^{45}$, has been upgraded to simulate solar system planet paleoclimates ${ }^{17,46-49}$ and exoplanet climates $^{50-52}$. The model uses the 3D dynamical core LMDz, which is based on a finitedifference formulation of the primitive equations of geophysical fluid dynamics. The GCM includes an up-to-date generalized radiative transfer (described in 'Radiative transfer in $\mathrm{H}_{2} \mathrm{O}$ rich atmospheres') that takes into account the absorption and scattering by the atmosphere, the clouds and the surface. The GCM includes condensation, evaporation, sublimation, and precipitation of water $\left(\mathrm{H}_{2} \mathrm{O}\right)$ (described in 'Turbulence, convection and clouds in $\mathrm{H}_{2} \mathrm{O}$-rich atmosphere'). For the present study, special care has been taken (see hereafter) to treat properly the situations in which the atmosphere is hot, thick and water-vapour-dominated. More details on the model can be found in refs ${ }^{19,50,52}$ (and references therein).

Our baseline simulations were computed at a horizontal resolution of $64 \times 48$ grid points (in longitude $\times$ latitude). In the vertical direction, the model is composed of 34 distinct atmospheric layers (using $\sigma$ hybrid coordinates, where $\sigma$ is the ratio between pressure and surface pressure) for the baseline simulations (between 31 and 40 for sensitivity studies), ranging from the surface up to $\sim 10 \mathrm{~Pa}$. The dynamical (for example, to compute winds) time step of the simulations is about $40 \mathrm{~s}$; the physical (for example, to compute convection) time step is about $6 \mathrm{~min}$; the radiative (for example, to compute radiative transfer) time step is about $1 \mathrm{~h}$.

Simulation input parameters for our baseline and sensitivity GCM simulations are summarized in Extended Data Table 1. The initial state of the simulations and the convergence method are described in 'A numerical scheme to accelerate the convergence of simulations'.

\section{Radiative Transfer in $\mathrm{H}_{2} \mathrm{O}$-rich atmospheres}

Our climate model includes a generalized radiative transfer code based on the correlated-k method $^{53}$ suited for fast calculations. We adapted the radiative transfer to any mixture of nitrogen $\left(\mathrm{N}_{2}\right)$ and $\mathrm{H}_{2} \mathrm{O}$ gases (as well as $\mathrm{CO}_{2}$ and $\mathrm{H}_{2} \mathrm{O}$ gases for sensitivity studies, as in $\mathrm{ref}^{49}$ ). 
Our radiative transfer calculations are performed on 55 spectral bands in the thermal infrared (from 0.65 to $100 \mu \mathrm{m}$ ) and 45 in the visible domain (from 0.3 to $6.5 \mu \mathrm{m}$ ). The infrared channel has been extended to relatively short wavelengths $(0.65 \mu \mathrm{m})$ to capture the thermal emission of the lowest and warmest (more than about $10^{3} \mathrm{~K}$ in some simulations) layers. Sixteen nonregularly spaced grid points were used for the $g$-space integration, where $g$ is the cumulative distribution function of the absorption for each band. $\mathrm{N}_{2}-\mathrm{N}_{2}{ }^{54}, \mathrm{H}_{2} \mathrm{O}-\mathrm{H}_{2} \mathrm{O}^{55}$ and $\mathrm{H}_{2} \mathrm{O}-\mathrm{N}_{2}{ }^{55}$ (using MT CKD version 3.2, from $200 \mathrm{~K}$ to $1,500 \mathrm{~K}$ and from 0 to $20,000 \mathrm{~cm}^{-1}$ ) continua were accounted for. Correlated-k tables were computed as in $\operatorname{ref}^{19}$ for $\mathrm{N}_{2}$ and $\mathrm{H}_{2} \mathrm{O}$ (and as in $\mathrm{ref}^{49}$ for $\mathrm{CO}_{2}$ and $\mathrm{H}_{2} \mathrm{O}$, in sensitivity studies), using the HITRAN $2008^{56}$ database for wavelength above $1 \mu \mathrm{m}$ and the HITEMP $2010^{57}$ database below $1 \mu \mathrm{m}$, following the results of $\operatorname{ref}^{28}$.

We performed 1D cloud-free radiative-convective model calculations ${ }^{29}$ using the radiative transfer parameterizations described above and found that the planetary albedo and thermal emission asymptotic limits calculations match very closely that of state-of-the-art $1 \mathrm{D}$ calculations of refs ${ }^{27,28}$ (see Extended Data Fig. 3). This validates the choices made for our radiative transfer calculations, which is used as is in the $3 \mathrm{D}$ simulations presented in this study.

The exact temperature at which the thermal emission departs from the asymptote depends very strongly on the water content in the atmosphere ${ }^{29}$. This is why we only compare here the values of the asymptote limit of refs $^{27,28}$ which have been shown to be weakly dependent on the total water content ${ }^{29}$.

\section{Turbulence, convection and clouds in $\mathrm{H}_{2} \mathrm{O}$-rich atmospheres}

Subgrid-scale dynamical processes (turbulent mixing and convection) were parameterized as in $\operatorname{ref}^{19}$. The planetary boundary layer was accounted for by the time-dependent 2.5-level closure scheme ${ }^{58,59}$, and complemented by a convective adjustment which rapidly mixes the atmosphere in the case of unstable temperature profiles. Moist convection was taken into account following a moist convective adjustment scheme that originally derives from the 'Manabe scheme' ${ }^{47,60}$. In the version of our scheme, relative humidity is let free and limited to $100 \%$ (supersaturation is not permitted). In practice, when an atmospheric grid cell reaches $100 \%$ saturation and the corresponding atmospheric column has an unstable temperature vertical profile, the moist convective adjustment scheme is performed to get a stable moist adiabatic lapse rate. We used a generalized formulation of the moist-adiabiat lapse rate 
developed in $\operatorname{ref}^{19}$ to account for the fact that water vapour is both the dominant and the condensible gas in our simulations. We also used the numerical scheme proposed in ref $^{19}$ to account for atmospheric mass change after the condensation or the evaporation of water vapour.

When condensing, water vapour form liquid water droplets or water ice particles, depending on the atmospheric temperature and pressure ${ }^{49,61}$, forming clouds. We used a fixed number of activated cloud condensation nuclei (CCNs) per unit mass of air $N_{c}$ to determine the local $\mathrm{H}_{2} \mathrm{O}$ cloud particle sizes, based on the amount of condensed material. $N_{c}$ was taken to be constant everywhere in the atmosphere, and equal to $10^{5} \mathrm{~kg}^{-1}$ for both liquid and ice water clouds in the baseline simulations (but varied in sensitivity simulations). This value roughly corresponds to that of high-altitude clouds on Earth ${ }^{62}$ and is also a standard value used for studies of rocky planet climates ${ }^{47,51,63}$.

The effective radius $r_{\text {eff }}$ of the cloud particles is then given by:

$$
r_{e f f}=\left(\frac{3 q_{c}}{4 \pi \rho_{c} N_{c}}\right)^{1 / 3}
$$

where $\rho_{c}$ is the density of the cloud particles $\left(1,000 \mathrm{~kg} / \mathrm{m}^{3}\right.$ for liquid and $920 \mathrm{~kg} / \mathrm{m}^{3}$ for water ice) and $q_{c}$ is the mass mixing ratio of cloud particles (in $\mathrm{kg}$ per $\mathrm{kg}$ of air). The effective radius of the cloud particles is then used to compute (1) their radiative properties calculated by Mie scattering and (2) their sedimentation velocity. Water precipitation is divided into rainfall and snowfall, depending on the nature (and thus the temperature) of the cloud particles. Rainfall is parameterised using the scheme of ref $^{64}$, accounting for the conversion of cloud liquid droplets to raindrops by coalescence with other droplets. Rainfall is considered to be instantaneous (that is, it goes directly to the surface) but can evaporate while falling through subsaturated layers. The evaporation rate of precipitation $E_{\text {precip }}\left(\mathrm{in} \mathrm{kg} / \mathrm{m}^{3} / \mathrm{s}\right.$ ) is determined by ${ }^{65}$ :

$$
E_{\text {precip }}=2 \times 10^{-5}\left(1-\frac{q_{v}}{q_{s, v}}\right) \sqrt{F_{\text {precip }}}
$$

where $q_{v}$ and $q_{s, v}$ are the water vapour mixing ratios in the air cell and at saturation, respectively. $F_{\text {precip }}$ is the precipitation flux (in $\mathrm{kg} / \mathrm{m}^{2} / \mathrm{s}$ ). In hot and steamy simulations, re-evaporation of precipitation is always complete, that is, precipitation always fully evaporate in the dry lower atmosphere before it reaches the ground. The snowfall rate is calculated using the sedimentation 
velocity of particles $V_{\text {sedim }}($ in $\mathrm{m} / \mathrm{s}$ ), assumed to be equal to the terminal velocity that we approximate by a Stokes law, with a 'slip-flow' correction factor ${ }^{66}$.

\section{A numerical scheme to accelerate the convergence of simulations}

The very high opacity and heat capacity of multi-bar water-dominated atmospheres considerably increases the computing time required for simulations to reach top-of-atmosphere radiative equilibrium. To overcome this issue, we implemented a numerical scheme designed to accelerate the convergence of GCM simulations towards equilibrium.

We start first with the GCM simulations at highest insolations $\left(675\right.$ and $\left.500 \mathrm{~W} / \mathrm{m}^{2}\right)$ with a partial pressure of $\mathrm{H}_{2} \mathrm{O}$ and $\mathrm{N}_{2}$ of 10 bar and 1 bar (in the baseline simulations), respectively, and an isothermal atmospheric temperature profile at 1,050 K. Although the upper atmospheric layers evolve very rapidly towards equilibrium, we had to implement a new strategy to converge the deepest layers of the atmosphere. Following ref $^{63}$, we artificially multiplied the heating rates (in shortwave and longwave channels) of the radiative transfer by a factor $\left(\frac{P}{P_{\text {lim }}}\right)^{\alpha}$ for atmospheric pressures $P>P_{\text {lim }}$, with $P_{\text {lim }}=10^{4} \mathrm{~Pa}$ and $\alpha$ a convergence factor. As the simulations get closer to convergence, we decrease the value of $\alpha$ from 0.5 to 0.3 and eventually to 0 . We consider that the simulations have reached their final equilibrium state when the topof-the-atmosphere radiative imbalance is typically lower than about $1 \mathrm{~W} / \mathrm{m}^{2}$.

We repeated the same procedure for simulations at lower insolations, except that we used the final equilibrium state of simulations at higher insolations as the initial state (see Extended Data Fig. 1, which shows the temporal evolution of the hot and steamy Venus simulations at several insolations).

On the whole, and taking advantage of the convergence scheme presented above, we have managed to converge all the simulations presented in this work using a total of around 600 kh CPU (central processing unit) on the French supercomputer OCCIGEN. Calculating the threshold insolation at which water condenses on the surface (which requires running simulations at multiple insolations) required around $150 \mathrm{kh}$ CPU for each configuration explored. 
To check that our accelerated convergence scheme is working effectively, we have performed several convergence tests (for the simulation of hot and steamy Venus, at an insolation of $500 \mathrm{~W} / \mathrm{m}^{2}$ ). We performed first a simulation in which we started with an isothermal atmospheric profile at $700 \mathrm{~K}$, and the simulation converged (less quickly though) towards the same final equilibrium. We have also performed two GCM simulations in which we started with a cold adiabatic profile $\left(\mathrm{T}_{\text {surf }}=700 \mathrm{~K}\right)$ and a hot adiabatic profile $\left(\mathrm{T}_{\text {surf }}=1,400 \mathrm{~K}\right)$ calculated with a $1 \mathrm{D}$ reverse numerical model ${ }^{29}$. Again, the simulations converged towards the same final equilibrium. Eventually, we have performed a last simulation in which we tested an alternative convergence scheme. After starting from an isothermal atmospheric profile at 1,050 $\mathrm{K}$, we proceeded with the following repeated steps:

1. We run the GCM for two Venusian days.

2. We extrapolate the evolution of the temperature field at time $t$ over $n_{\text {days }}$ (the number of Venusian days) using:

$$
T_{i, j, k}\left(t+n_{\text {days }}\right)=T_{i, j, k}(t)+n_{\text {days }} \times \Delta T_{\text {mean }, k}
$$

with $T_{i, j, k}$ the temperature at the $i, j, k$ spatial coordinates (corresponding respectively to longitude, latitude and altitude coordinates) and $\Delta T_{\text {mean }, k}$ the change (over the second Venusian day of GCM simulations) of the mean horizontal (averaged over all longitudes and latitudes) temperature field at the altitude layer $k$. $n_{\text {days }}$ was first set arbitrarily to 50 , then 10 and eventually 0 , when the planetary atmosphere is close to convergence. We limit the variations of temperature to $50 \mathrm{~K}$ to avoid numerical instabilities.

3. We repeat the previous steps, until the top-of-the-atmosphere radiative imbalance is lower than about $1 \mathrm{~W} / \mathrm{m}^{2}$.

With this alternative convergence scheme, the simulation converged (less quickly though) towards the same final equilibrium. These sensitivity tests validate the use of our accelerated convergence scheme. 


\section{Comparisons with previous 1D modelling}

We first validated our radiative transfer parameterizations and calculations by comparing the results of our cloud-free 1D numerical climate calculations ${ }^{29}$ with that of refs ${ }^{27,28}$. Extended Data Figure 3 shows that the moist tropospheric asymptotic limit - sometimes also known as the Simpson- Nakajima limit ${ }^{67-69}$ - matches closely that of $\operatorname{refs}^{27,28}$.

Extended Data Figure 3 also shows comparisons with the results of our baseline 3D GCM simulations of hot and steamy Earth and Venus. Differences with 1D calculations arise from the effects of dynamics, convection, radiation and mostly clouds. The dayside cloud coverage decreases with increasing insolation (and thus, the surface temperature), which is reflected in the evolution of the bond albedo with surface temperature. For the highest insolation explored in this study (about $675 \mathrm{~W} / \mathrm{m}^{2}$, corresponding to a surface temperature of $\sim 1,060 \mathrm{~K}$ for Earth and Venus, in Extended Data Fig. 3b), the bond albedo of 3D simulations is very close to that predicted with state-of-the-art 1D cloud-free calculations ${ }^{27-29}$.

Thermal emission to space is significantly different from that predicted with 1D cloudfree calculations for two reasons. First, nightside clouds are always present for the range of insolations $\left(\sim 325-675 \mathrm{~W} / \mathrm{m}^{2}\right)$ explored in this study. These clouds produce a strong greenhouse effect that significantly reduces thermal emission (Extended Data Fig. 2). This is well visible at low insolation (or at the lowest surface temperatures) in Extended Data Fig. 3a. Second, the 1D reverse calculations ${ }^{27-29}$ assume fixed temperature profiles (that is, with fixed stratospheric temperatures, at $200 \mathrm{~K}$, and convective profiles otherwise; this is illustrated by the green profile in Fig. 3b). Our 3D baseline calculations do take into account the direct warming of the atmosphere by the incoming solar flux, which produces significant heating at the subsolar point (see Fig. 3d), and thus higher stratospheric temperatures at the subsolar point (see Fig. 3b), which produces a rapid increase (compared with $1 \mathrm{D}$, reverse models) of the thermal emission to space with insolation (see Extended Data Fig. 3a). This effect could be implemented in 1D reverse numerical climate models by implementing a parameterisation of the vertical temperature structure as a function of the insolation.

\section{Sensitivity studies}

We proceeded to several sensitivity studies to test the robustness of the nightside cloud feedback warming mechanism. 


\section{i. varying cloud microphysical properties}

We performed a first sensitivity study (for hot and steamy Venus, insolation at $500 \mathrm{~W} / \mathrm{m}^{2}$, that is, that of Venus about 4 billion years ago) in which we varied the number of Cloud Condensation Nuclei (CCN), the key parameter ${ }^{46,63}$ of the clouds microphysics model used in our GCM, for a wide range of values from $10^{3}$ to $10^{7}$ per $\mathrm{kg}$ of atmosphere. The results, which are summarized in Extended Data Fig. 4, reveal that the dichotomic structure of the clouds (absence of clouds on the dayside, presence of clouds on the nightside) and the net warming effect of clouds are robust to the choice of the number of CCNs. The net radiative effect of clouds increases with the number of CCNs. It is equal to $20,58,98,104$ and $108 \mathrm{~W} / \mathrm{m}^{2}$ for a CCN number of $10^{3}, 10^{4}, 10^{5}, 10^{6}, 10^{7} \mathrm{~kg}^{-1}$, respectively.

\section{ii. varying the amount of water}

Our baseline 3D GCM simulations of hot and steamy Earth and Venus were computed assuming a $\mathrm{H}_{2} \mathrm{O}$ partial pressure of $10 \mathrm{bar}$, corresponding to around $100 \mathrm{~m}$ GEL on Earth and $110 \mathrm{~m}$ GEL on Venus. We performed a sensitivity study (for hot and steamy Venus, insolation at $500 \mathrm{~W} / \mathrm{m}^{2}$ ) in which we varied the $\mathrm{H}_{2} \mathrm{O}$ partial pressure between 1 bar and 30 bar, corresponding to around 11-330 m GEL on Venus. Simulations at 30 bar of $\mathrm{H}_{2} \mathrm{O}$ show very similar behaviour to those at $10 \mathrm{bar}$, which results from the fact that the pressure is high enough to decouple the surface from the upper part of the atmosphere. We therefore expect that simulations with a partial pressure of $\mathrm{H}_{2} \mathrm{O}$ larger than 30 bar (that is, GEL larger than around $300 \mathrm{~m}$ ) also show a similar behaviour. This means our simulations are roughly representative of the range of past water content estimates on Venus ${ }^{4}$.

Extended Data Fig. 5 summarizes the results of this sensitivity study. The net warming effect of clouds is similar across simulations. We also observe, as predicted in 1D numerical climate models ${ }^{29}$, that the surface temperature increases with $\mathrm{H}_{2} \mathrm{O}$ partial pressure. The bond albedo and thermal emission are relatively stable across simulations.

\section{iii. adding carbon dioxide}

Our baseline 3D GCM simulations of hot and steamy Earth and Venus were computed assuming a $\mathrm{N}_{2}$ partial pressure of 1 bar. 
We have performed two sensitivity experiments in which $\mathrm{N}_{2}$ was replaced by 1 bar and 10 bar of $\mathrm{CO}_{2}$. We carried out these experiments to take into account the possibility that $\mathrm{CO}_{2}$ was present in large quantities in the atmosphere of early Venus ${ }^{70}$ as it is today. Here again, we observe that clouds preferentially form on the nightside, having thus a net warming effect (see Extended Data Fig. 5).

We observe that the bond albedo increases with $\mathrm{CO}_{2}$ partial pressure, as predicted by previous $\mathrm{CO}_{2}+\mathrm{H}_{2} \mathrm{O}$ 1D modeling ${ }^{71}$, due to $\mathrm{CO}_{2}$ having a larger Rayleigh scattering crosssection than $\mathrm{H}_{2} \mathrm{O}$. The bond albedo is lower than that of 1D cloud-free $\mathrm{CO}_{2}+\mathrm{H}_{2} \mathrm{O}$ simulations of ref ${ }^{71}$, not only because the 3D GCM simulations are depleted in dayside clouds, but also because some visible water lines - present in HITEMP ${ }^{57}$ and not in HITRAN ${ }^{56}$, which was used in ref $^{71}$ - increase the absorption, further lowering the albedo ${ }^{28}$.

\section{iv. influence of the initial cloud cover distribution}

We performed a last sensitivity study (for hot and steamy Venus, insolation at $500 \mathrm{~W} / \mathrm{m}^{2}$ ) in which we artificially forced the cloud cover to be complete on both the night and day sides, in order to explore whether clouds on the dayside could push the planet towards cooling and thus ultimately surface water condensation. The sensitivity simulation shows that the cloud distribution very quickly regains a dichotomous structure after a few Earth days of simulation only.

\section{ACKNOWLEDGEMENTS}

This project has received funding from the European Union's Horizon 2020 research and innovation program under the Marie Sklodowska-Curie Grant Agreement No. 832738/ESCAPE. This project has received funding from the European Research Council (ERC) under the European Union's Horizon 2020 research and innovation program (grant agreements No. 724427/FOUR ACES and 679030/WHIPLASH). This work has been carried out within the framework of the National Centre of Competence in Research PlanetS supported by the Swiss National Science Foundation. We acknowledge the financial support of the SNSF. M.T. thanks the Gruber Foundation for its support to this research. M.T. thanks N. Chaniaud for her help in preparing Fig. 1. We thank the LMD Generic global climate model team for the 
teamwork development and improvement of the model. This work was performed using the High-Performance Computing (HPC) resources of Centre Informatique National de l'Enseignement Supérieur (CINES) under the allocation A0080110391 made by Grand Équipement National de Calcul Intensif (GENCI). A total of about 600,000 CPU hours were used for this project on the OCCIGEN supercomputer, resulting in roughly one ton equivalent of $\mathrm{CO}_{2}$ emissions.

\section{DATA AVAILABILITY}

The data that support the findings of this study are available at https://doi.org/10.5281/zenodo.4680905. Source data are provided with this paper.

\section{CODE AVAILABILITY}

The LMD Generic global climate model code (and documentation on how to use the model) used in this work can be downloaded from the SVN repository https://svn.lmd.jussieu.fr/Planeto/trunk/LMDZ.GENERIC/ (version 2528). More information and documentation are available on http://www-planets.lmd.jussieu.fr.

\section{AUTHOR CONTRIBUTIONS}

M.T. developed the core ideas of the manuscript, developed and performed the 3D GCM simulations, wrote the manuscript and prepared the figures. E.B. and G.C. provided advice on sensitivity studies. D.E., G.C. and J.L. provided advice on the structure of the figures. J.L. provided advice on the organization of the manuscript, as well as for understanding the mechanism of cloud formation. E.M. provided advice on literature selection. All authors provided guidance and comments on the manuscript. 


\section{COMPETING INTERESTS}

The authors declare that they have no competing financial interests.

\section{CORRESPONDENCE}

Correspondence and requests for materials should be addressed to M.T.

(martin.turbet@unige.ch).

\section{REFERENCES}

44. Hourdin, F. et al. The LMDZ4 general circulation model: climate performance and sensitivity to parametrized physics with emphasis on tropical convection. Climate Dynamics 27, 787-813 (2006).

45. Forget, F. et al. Improved general circulation models of the Martian atmosphere from the surface to above $80 \mathrm{~km}$. Journal of Geophysical Research 104, 24155-24176 (1999).

46. Forget, F. et al. 3D modelling of the early Martian climate under a denser $\mathrm{CO}_{2}$ atmosphere: Temperatures and $\mathrm{CO}_{2}$ ice clouds. Icarus 222, 81-99 (2013).

47. Wordsworth, R. et al. Global modelling of the early Martian climate under a denser $\mathrm{CO}_{2}$ atmosphere: Water cycle and ice evolution. Icarus 222, 1-19 (2013).

48. Turbet, M., Forget, F., Leconte, J., Charnay, B. \& Tobie, G. $\mathrm{CO}_{2}$ condensation is a serious limit to the deglaciation of Earth-like planets. Earth and Planetary Science Letters 476, 11-21 (2017).

49. Turbet, M. et al. The environmental effects of very large bolide impacts on early Mars explored with a hierarchy of numerical models. Icarus 335, 113419 (2020).

50. Wordsworth, R. D. et al. Gliese 581d is the First Discovered Terrestrial-mass Exoplanet in the Habitable Zone. The Astrophysical Journal Letters 733, L48 (2011).

51. Leconte, J. et al. 3D climate modeling of close-in land planets: Circulation patterns, climate moist bistability, and habitability. Astronomy \& Astrophysics 554, A69 (2013).

52. Turbet, M. et al. Modeling climate diversity, tidal dynamics and the fate of volatiles on TRAPPIST-1 planets. Astronomy \& Astrophysics 612, A86 (2018).

53. Fu, Q. \& Liou, K. N. On the correlated k-distribution method for radiative transfer in nonhomogeneous atmospheres. Journal of Atmospheric Sciences 49, 2139-2156 (1992).

54. Karman, T. et al. Update of the HITRAN collision-induced absorption section. Icarus 328, 160-175 (2019).

55. Mlawer, E. J. et al. Development and recent evaluation of the MT CKD model of continuum absorption. Philosophical Transactions of the Royal Society of London Series A 370, 2520- 2556 (2012).

56. Rothman, L. S. et al. The HITRAN 2008 molecular spectroscopic database. Journal of quantitative spectroscopy \& Radiative Transfer 110, 533-572 (2009).

57. Rothman, L. S. et al. HITEMP, the high-temperature molecular spectroscopic database. Journal of quantitative spectroscopy \& Radiative Transfer 111, 2139-2150 (2010).

58. Mellor, G. L. \& Yamada, T. Development of a turbulence closure model for geophysical fluid problems. Reviews of Geophysics and Space Physics 20, 851-875 (1982). 
59. Galperin, B., Kantha, L. H., Hassid, S. \& Rosati, A. A Quasi-equilibrium Turbulent Energy Model for Geophysical Flows. Journal of Atmospheric Sciences 45, 55-62 (1988).

60. Manabe, S. \& Wetherald, R. Thermal equilibrium of the atmosphere with a given distribution of relative humidity. Journal of Atmospheric Sciences 24, 241-259 (1967).

61. Charnay, B. Tropospheric dynamics and climatic evolution of Titan and the early Earth. Theses, Université Pierre et Marie Curie - Paris VI (2014). URL https://tel.archivesouvertes.fr/tel-00987546.

62. Wallace, J. M. \& Hobbs, P. V. 6 - cloud microphysics. In Wallace, J. M. \& Hobbs, P. V. (eds.) Atmospheric Science (Second Edition), 209-269 (Academic Press, San Diego, 2006), second edition edn. URL https://www.sciencedirect.com/science/article/pii/B9780127329512500119.

63. Charnay, B. et al. Formation and dynamics of water clouds on temperate sub-Neptunes: the example of K2-18b. Astronomy \& Astrophysics 646, A171 (2021).

64. Boucher, O., Le Treut, H. \& Baker, M. B. Precipitation and radiation modeling in a general circulation model: Introduction of cloud microphysical processes. Journal of Geophysical Research 100, 16 (1995).

65. Gregory, D. A Consistent Treatment of the Evaporation of Rain and Snow for Use in Large- Scale Models. Monthly Weather Review 123, 2716 (1995).

66. Rossow, W. B. Cloud microphysics - Analysis of the clouds of Earth, Venus, Mars, and Jupiter. Icarus 36, 1-50 (1978).

67. Nakajima, S., Hayashi, Y.-Y.\& Abe, Y. A study on the 'runaway greenhouse effect' with a one-dimensional radiative-convective equilibrium model. Journal of Atmospheric Sciences 49, 2256-2266 (1992).

68. Goldblatt, C. \& Watson, A. J. The runaway greenhouse: implications for future climate change, geoengineering and planetary atmospheres. Philosophical Transactions of the Royal Society of London Series A 370, 4197-4216 (2012).

69. Marcq, E., Salvador, A., Massol, H. \& Davaille, A. Thermal radiation of magma ocean planets using a 1-D radiative-convective model of $\mathrm{H}_{2} \mathrm{O}-\mathrm{CO}_{2}$ atmospheres. Journal of Geophysical Research (Planets) 122, 1539-1553 (2017).

70. Massol, H. et al. Formation and Evolution of Protoatmospheres. Space Science Reviews 205, 153-211 (2016).

71. Pluriel, W., Marcq, E. \& Turbet, M. Modeling the albedo of Earth-like magma ocean planets with $\mathrm{H}_{2} \mathrm{O}-\mathrm{CO}_{2}$ atmospheres. Icarus 317, 583-590 (2019). 


\begin{tabular}{|c|c|c|}
\hline Physical Parameters & Values (Earth) & Values (Venus) \\
\hline Solar Flux $\left(\mathrm{W} / \mathrm{m}^{2}\right)$ & $312.5,325$, & $375,500,675$ \\
\hline Obliquity $\left(^{\circ}\right)$ & 23.5 & 177 \\
\hline Orbital eccentricity & \multicolumn{2}{|c|}{0} \\
\hline Rotation period (h) & 24 & 5,833 \\
\hline Solar day (h) & 24 & 2,802 \\
\hline Radius (km) & 6,370 & 6,052 \\
\hline Gravity $\left(\mathrm{m} / \mathrm{s}^{2}\right)$ & 9.81 & 8.87 \\
\hline Bare ground albedo & \multicolumn{2}{|c|}{0.2} \\
\hline $\begin{array}{l}\text { Ground thermal inertia } \\
\qquad\left(\mathrm{J} / \mathrm{m}^{2} / \mathrm{s}^{1 / 2} / \mathrm{K}\right)\end{array}$ & \multicolumn{2}{|c|}{2,000} \\
\hline Surface topography & \multicolumn{2}{|c|}{ flat } \\
\hline $\begin{array}{l}\text { Surface roughness } \\
\text { coefficient }(\mathrm{m})\end{array}$ & \multicolumn{2}{|c|}{0.01} \\
\hline $\mathrm{H}_{2} \mathrm{O}$ partial pressure (bar) & 10 & $10 *$ \\
\hline $\mathrm{N}_{2}$ partial pressure (bar) & 1 & $1^{\dagger}$ \\
\hline $\mathrm{CO}_{2}$ partial pressure (bar) & 0 & $0 *$ \\
\hline $\begin{array}{l}\text { Number of } \mathrm{H}_{2} \mathrm{O} \text { cloud } \\
\text { condensation nuclei }(\mathrm{CCN}) \\
\text { for ice and liquid }\left(\mathrm{kg}^{-1}\right)\end{array}$ & $10^{5}$ & $10^{5 \S}$ \\
\hline
\end{tabular}

Extended Data Table 1 | Summary of the main physical parameters used in the GCM for the baseline hot and steamy Earth and Venus simulations. * We varied $\mathrm{H}_{2} \mathrm{O}$ partial pressure from 1 to 30 bar in some hot and steamy Venus GCM sensitivity simulations; ${ }^{\dagger} \mathrm{We}$ fixed $\mathrm{N}_{2}$ partial pressure to 0 bar in hot and steamy Venus GCM sensitivity simulations with $\mathrm{CO}_{2}$; In hot and steamy Venus GCM sensitivity simulations with $\mathrm{CO}_{2}$, we used partial pressure of 1 and 10 bar of $\mathrm{CO}_{2} ;{ }^{\S} \mathrm{We}$ varied the number of $\mathrm{CCN}$ from $10^{3}$ to $10^{7}$ in GCM sensitivity simulations of Venus. 


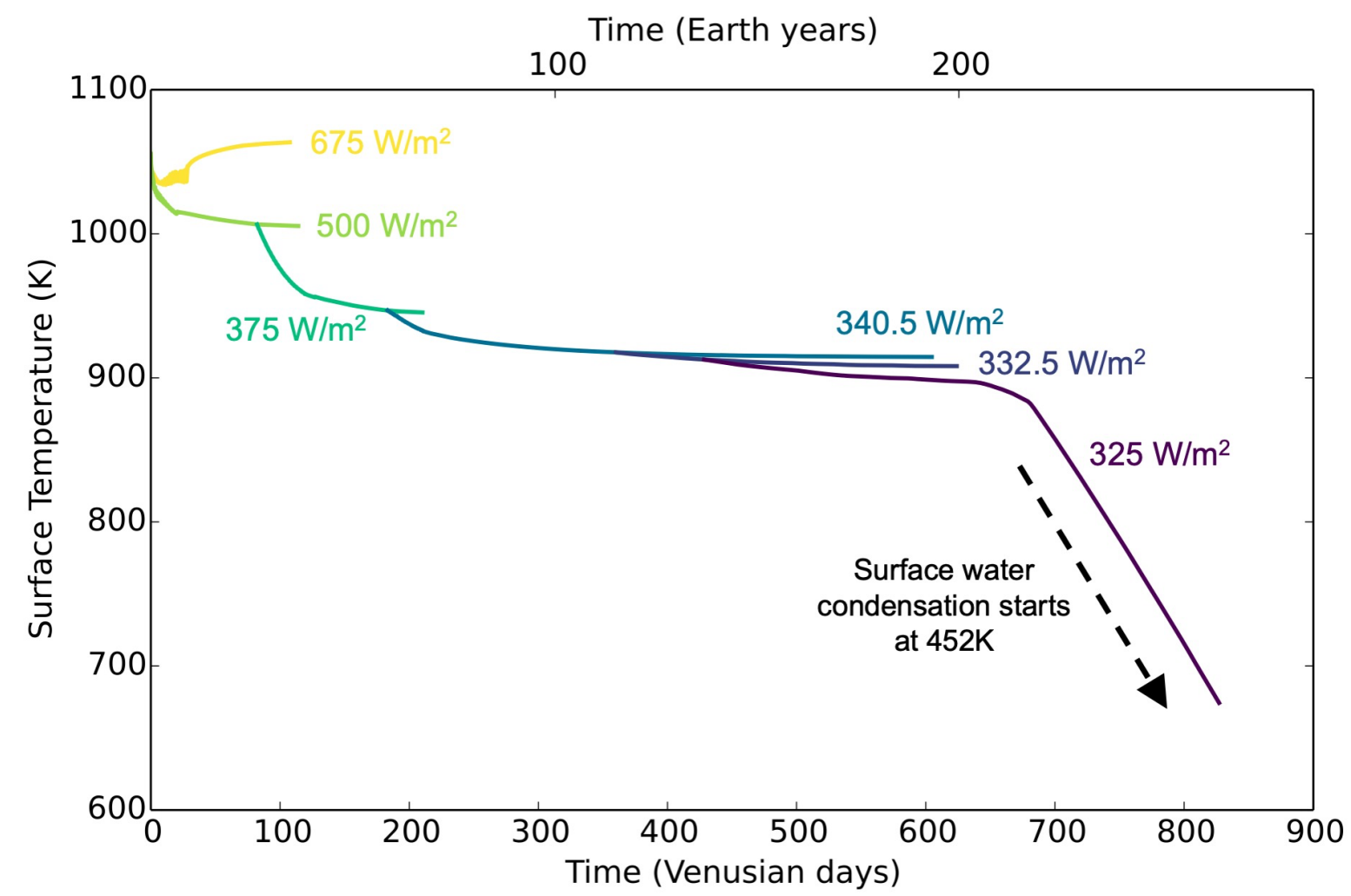

Extended Data Figure 1 | Temporal evolution of modelled surface temperatures.

Temporal evolution of the globally-averaged surface temperatures in the 3D GCM baseline simulations of (initially hot and steamy) Venus, for several insolations. 

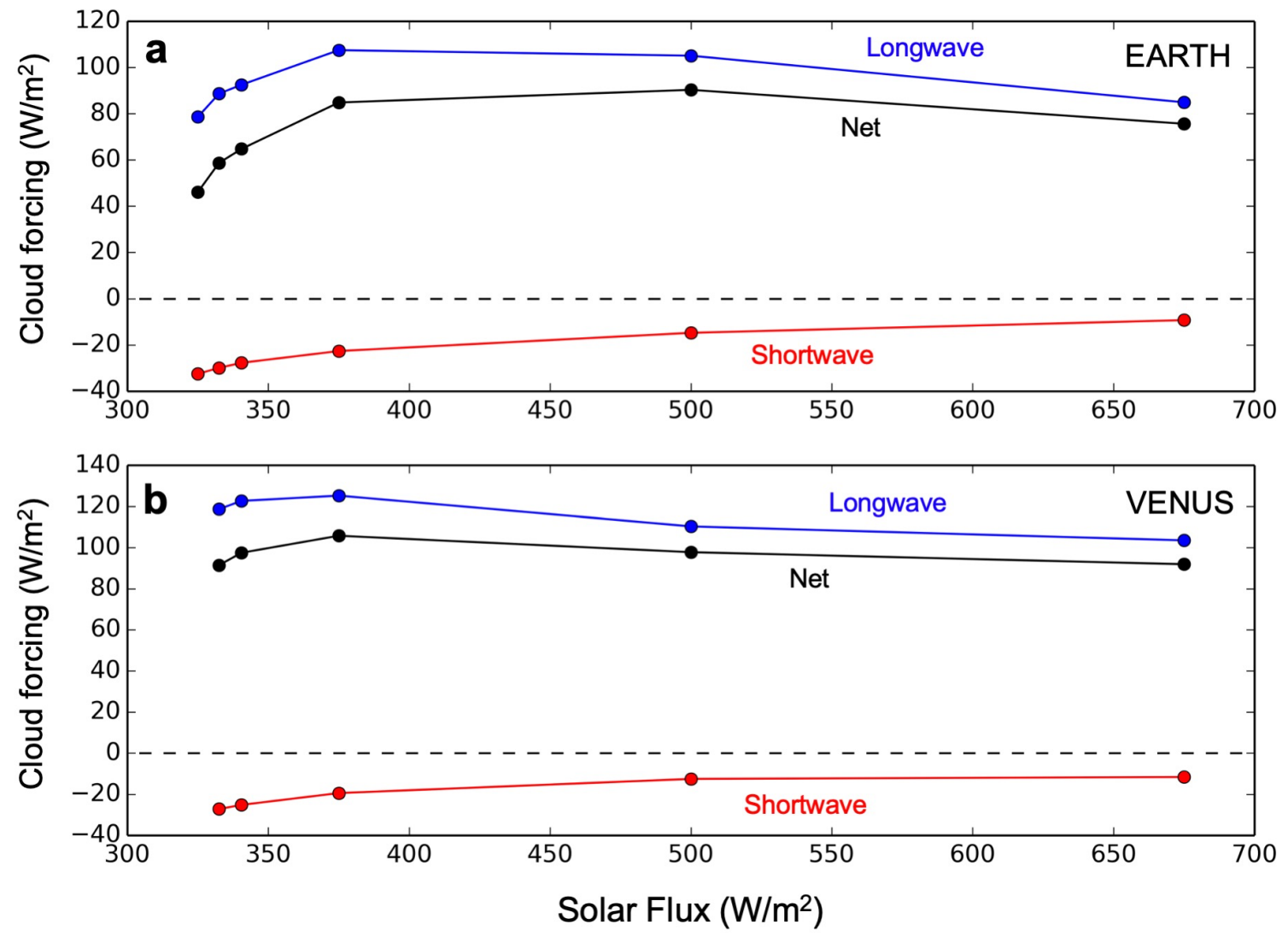

\section{Extended Data Figure 2 | Cloud forcings in hot and steamy early Venus and Earth}

simulations. Radiative balance of clouds on hot and steamy Earth (a) and Venus (b) as a function of the incident solar flux. Blue curves indicate the greenhouse effect of clouds. Red curves indicate the amount of incoming solar radiation reflected back by the clouds (the more negative the value, the greater the reflected flux.). Black curves indicate the net radiative effect of clouds (positive values mean warming). In all initially hot and steamy simulations, clouds lead to a strong atmospheric warming. 

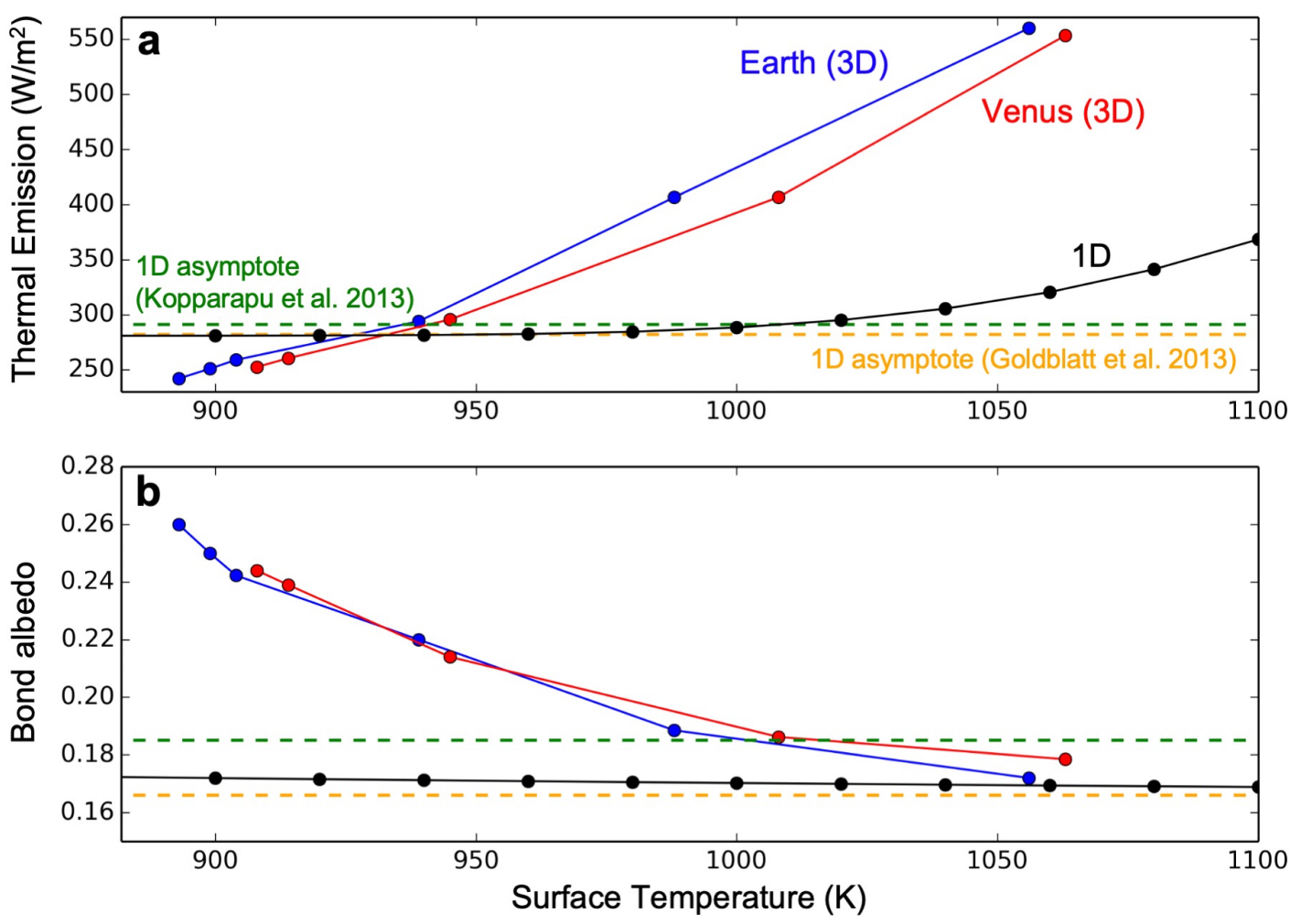

Extended Data Figure 3 | Radiative budget comparisons between 1D and 3D models.

Thermal emission to space (a) and bond albedo (b) as a function of the surface temperature for our 3D GCM simulations of Earth (blue) and Venus (red). We have also added the results of $1 \mathrm{D}$ radiative-convective cloud-free calculations ${ }^{29}$ (in black), using $\mathrm{H}_{2} \mathrm{O}$ and $\mathrm{N}_{2}$ partial pressures of 10 and 1 bar, respectively, as in the 3D baseline simulations. For comparison, we added the moist tropospheric radiation limits ${ }^{68}$ for the thermal emission ${ }^{67}$ and the bond albedo from refs ${ }^{27,28}$ 

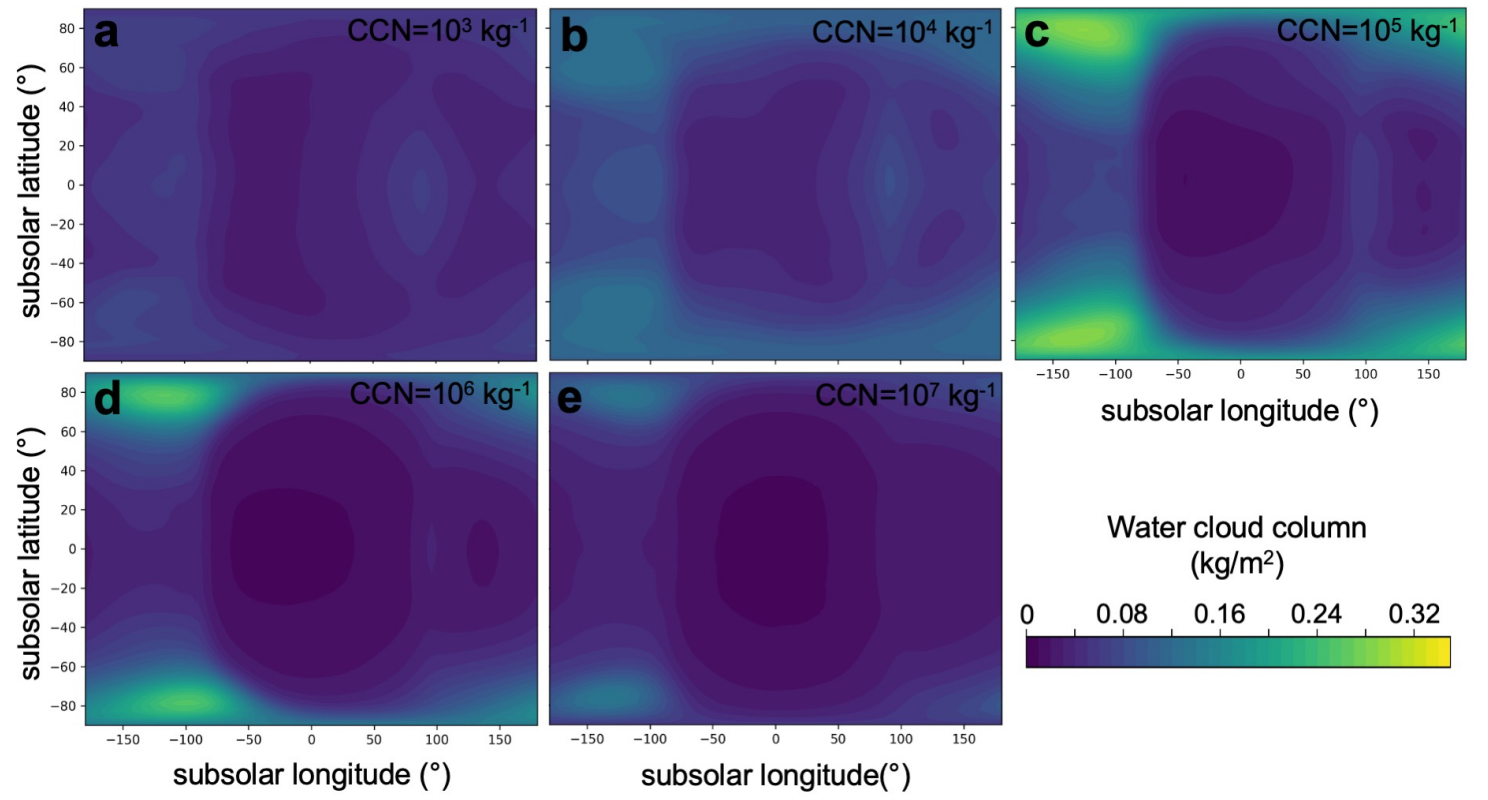

subsolar longitude $\left({ }^{\circ}\right)$

Water cloud column $\left(\mathrm{kg} / \mathrm{m}^{2}\right)$

$\begin{array}{lllll}0 & 0.08 & 0.16 & 0.24 & 0.32\end{array}$

Extended Data Fig. 4 | Impact of cloud microphysical properties on their spatial

distribution. Maps of water cloud column for early Venus (at a top-of-atmosphere insolation of $500 \mathrm{~W} / \mathrm{m}^{2}$, i.e. the minimal insolation received on Venus, about 4 billion years ago when the Sun was $25 \%$ fainter than today), with different cloud microphysics parameterisations $\left(10^{3}, 10^{4}, 10^{5}, 10^{6}\right.$ and $10^{7}$ Cloud Condensation Nuclei $(\mathrm{CCN})$ per kg of air, for panels a, b, c, $\mathrm{d}$ and e, respectively). The maps were calculated in the heliocentric frame (i.e., keeping the subsolar point at $0^{\circ}$ longitude and $0^{\circ}$ latitude), and using an average of two Venusian days. The distribution of clouds (present on the nightside, absent on the dayside) is robust to the choice of the number of CCN. 

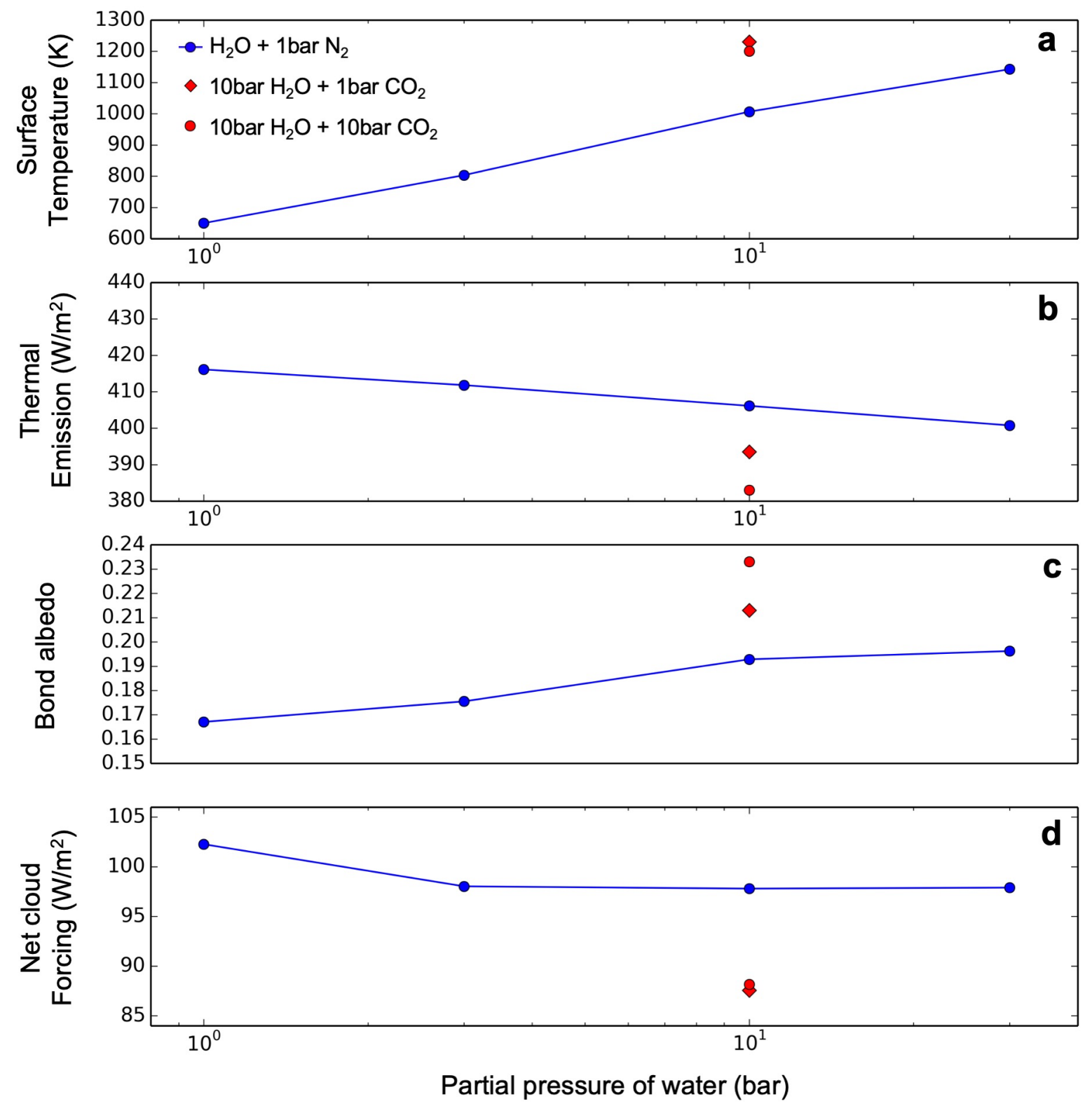

Extended Data Fig. 5 | Effects of water and carbon dioxide atmospheric contents. Impact of the water and carbon dioxide atmospheric contents on the surface temperature (a), thermal emission to space (b), bond albedo (c) and net cloud radiative forcing (d). The calculations assume a hot and steamy Venus (insolation at $500 \mathrm{~W} / \mathrm{m}^{2}$ ) with 1 bar of $\mathrm{N}_{2}$ and between 1 and 30 bar of $\mathrm{H}_{2} \mathrm{O}$ (in blue); with 1-10 bar of $\mathrm{CO}_{2}$ and 10 bar of $\mathrm{H}_{2} \mathrm{O}$ (in red). 